T. S. Gadjiev, Ya. Rustamov, S. Aliev, T. A. Maharramova

\title{
FORCING THE SYSTEM BY A DRIFT
}

T.S. Gadjiev, Ya. Rustamov, S. Aliev, T.A. Maharramova. Forcing the system by a drift, Mat. Stud. 55 (2021), 201-205.

We establish apriori estimate for the solutions of a degenerate non-divergence nonlinear elliptic equation. For this goal we study forcing the system by a drift.

We consider a nonlinear elliptic equation of non-divergence type

$$
\sum_{i, j=1}^{n} a_{i j}(x, u(x), D u(x)) D^{2} u(x)+f(x, u, D u(x))=0 .
$$

Let $B_{2 r} \subset \mathbb{R}^{n}$ be a ball with radius $2 r, r \geq 1$. The solution of equation (1) is searched from $C\left(\bar{B}_{2 r}\right) \cap W_{l o c}^{2, n}\left(B_{2 r}\right)$. Here $a_{i j}=a_{j i}$, i.d. correspondingly $A(x, y, p)$ is a symmetric matrix of size $n \times n$ and $\forall y \in R, \forall x, p, \xi \in \mathbb{R}^{n}$ coefficients satisfy

$$
\begin{gathered}
\Lambda^{-1} \lambda(p) \omega(x)|\xi|^{2} \leq(\xi, A(x, y, p) \xi) \leq \Lambda \lambda(p) \omega(x)|\xi|^{2} \\
f(x, y, p) \leq \frac{\Lambda}{k}(1+\lambda(p))(1+|p|)
\end{gathered}
$$

for some $\Lambda \geq 1, k>1$. Let $\lambda: \mathbb{R}^{n} \rightarrow \mathbb{R}_{+}$be a some continuous mapping for which there exists $\lambda_{0}$ and $M>0$ such that $\lambda(z) \geq \lambda_{0}$ for $|z| \geq M, \omega(x)$ is the Muckenhoupt weight function (see [3]). Let $u: \bar{B}_{2 r} \rightarrow \mathbb{R}$ be a bounded and continuous solution of (1).

The regularity estimates for a solution of divergence form equation were investigated by De Giorgi and Nash (see [7, 12]). Investigations by Serrin [13] and Ladyzhenskaya, Uraltsev [11], De Giorgi and Nash show that these estimates are valid for quasilinear elliptic equations of divergent type $[1,2,6,14])$. A corresponding result for the non-divergence equations was obtained by Krylov and Safanov. These authors used probability methods (see [9, 10]).

The strategy of the proof results for system (1) relies on a well-known probabilistic interpretation of the nonlinear equations. The proof consists in introducing a diffusion process $X$, solution to the stochastic differential equation (SDE)

$$
d X_{t}=\sigma\left(X_{t}, u\left(X_{t}\right) D u\left(X_{t}\right)\right) d W_{t}, \quad t \geqslant 0,
$$

where $W$ is a Wiener process and $\sigma$ is a contiuous version of the square root of the matricial mappings 2A. The basic idea follows from the theory of diffusion processes, the generator of a diffusion process enjoys some smoothing property if the path of the corresponding process sufficiently visit the surrounding space with a non trivial probability. The argument may be understood as follows: $U$ is smooth: in such a framework, $\left(u\left(X_{t}\right)\right)_{t \geqslant 0}$ is a martingale.

2010 Mathematics Subject Classification: 35J25, 35J70, 60H15.

Keywords: degenerate nonlinear elliptic equations; weighted Hölder estimate; stohastic differential equations. doi:10.30970/ms.55.2.201-205

(C) T. S. Gadjiev, Ya. Rustamov, S. Aliev, T. A. Maharramova, 2021 
In particular, $U(x)$ may be expressed as the expectation $E\left[u\left(X_{\tau}\right)\right]$ for any well-controlled stopping time $\tau$.

In the special probability theory, the point is to bound from below the probability that the diffusion process $X$ hits a Borel subset included in $B_{2}$, before leaving it. We specifically show that we can force the stochastic system on the areas of degeneracy by an additional drift to push it towards the desired Borel subset. When $|D u|$ is large by an ellipticity condition, the probability theory can be applied. Theorem 1 says that the probability of hitting a Borel subset $V$ of $Q_{\rho}$ before leaving the ball $Q_{\rho}$ is bounded from below by a constant only depending on $n$ and $\Lambda$ and on the proportion of $V$ in $Q_{\rho}$. The connection with system (1) may be understood as follows: when $u$ is strong solution of (1), we choose $a(x)$ in the statement of Theorem 1 as $2 A(x, u(x), D u(x))$. We deduce that $\left|D u\left(X_{t}\right)\right| \geqslant M$. In other words, the resulting drift $\left(b_{t}\right)_{t \geqslant 0}$ just acts when the gradient is small, i.e. bounded. Later we can use Theorem 1 and establish a priori Hölder estimate as in the result by Krylov and Safanov.

The goal of this paper is to prove a similar result for degenerate quasilinear elliptic equations of non-divergence form. Therefore firstly we study forcing the system by a drift.

Also, we suppose that the following condition is satisfied: let $\sigma: \mathbb{R}^{n} \rightarrow \mathbb{R}^{n \times n}$ be a Lipschitz continuous mapping such that $\forall x, \xi \in \mathbb{R}^{n}$

$$
\Lambda^{-1} \lambda(x) \omega(x)|\xi|^{2} \leq(\xi, a(x) \xi) \leq \Lambda \bar{\lambda}(x) \omega(x)|\xi|^{2},
$$

$a(x)=\sigma \sigma^{*}(x)$, for some $\Lambda \geq 1$ and some mapping $\bar{\lambda}: \mathbb{R}^{n} \rightarrow[0,1]$.

Let $\left(\Omega, F,\left(F_{t}\right)_{t \geq 0}, P\right)$ be a filtered probability space satisfying the usual conditions endowed with an $\left(F_{t}\right)_{t \geq 0}$ Brownian motion $\left(W_{t}\right)_{t \geq 0}, \alpha$ be a positive real and $Q_{1}$ be some hypercube of $\mathbb{R}^{n}$ of radius $1 ; Q_{\rho}$ is the hypercube of same center as $Q_{1}$ but of radius $\rho$; $Q(z, s)$ is the hypercube at the center $z$ and radius $\rho, Q(0,1) \equiv Q_{1}$.

Under these conditions on the coefficients, we can build a drift to force the system to hit a prescribed Borel subset of large measure with a non-zero probability.

Out the prof of results for system (1) relies on a probabilistic interpetation of the nonlinear PDE. We need the main Theorem 1. Later we choose $a(x)$ in the statement of Theorem 1 as $2 A(x, u(x), D u(x))$.

Theorem 1. Let $\sigma: \mathbb{R}^{n} \rightarrow \mathbb{R}^{n \times n}$ be a Lipschitz continuous mapping such that $a(x)=\sigma \sigma^{*}(x)$ satisfies the condition (3) and $\alpha \in(0,+\infty)$. Then, there exist positive constants $\mu_{0}, \varepsilon_{0}, R_{0}$ and $\left(\Gamma_{p}(\mu)\right)_{1 \leq p<2}$, only depending on $d, \alpha, \lambda, \omega$, such that, for any $\rho \in(0,1)$, any hypercubes $Q_{\rho / 8} \subset Q_{1} \subset \mathbb{R}^{n}$ and any square integrable $F_{0}$-measurable random variable $X_{0}$ with values in $\mathbb{R}^{n}$, we can find an integrable $n$-dimensional progressively-measurable process $\left(b_{t}\right)_{t \geq 0}$ and the process $X$, solution to the SDE

$$
X_{t}=X_{0}+\int_{0}^{t} b_{s} d s+\int_{0}^{t} \omega\left(X_{s}\right) \sigma\left(X_{s}\right) d W_{s}, t \geq 0,
$$

for which it fulfills $\forall t \geq 0, \lambda\left(X_{t}\right) \geq \alpha \Rightarrow b_{t}=0$, and also $E_{0} \int_{0}^{+\infty}\left|b_{t}\right|^{p} d t \geq \Gamma_{p} \rho^{p-2}$ $(\forall p \in[1,2))$. Moreover, for any Borel subset $V \subset Q_{\rho}$ one has

$$
\left|Q_{\rho} \backslash V\right| \leq \mu_{0}\left|Q_{\rho}\right| \Rightarrow P_{0}\left\{T_{V}<\left(R_{0} \rho^{2}\right) \cap S_{Q_{\rho}}\right\} \geq \varepsilon_{0},
$$

a.e. on the event $\left\{X_{0} \in Q_{\rho / 8}\right\}$. Here $T_{V}$ is the first hitting time of $V$ and $S_{Q_{\rho}}$ is the first exit time $Q_{\rho}$ by $X$. 
Proof. Let $\delta$ be a small real, for example, at least than $\frac{1}{4}$. By a scaling argument we take $\rho=1$. We mean some a function $X_{t}$ of $\delta$ that only depends on $d, \alpha, \Lambda, \omega$ and that tends to zero as $\delta$ tends to zero. We can assume that $X_{0} \in Q_{1 / 8}$ a.e., if need we can change the values of $X_{0}$. Also let $\left|Q_{1} \backslash V\right| \leq q_{0}$, where $q_{0}$ is a universal constant, only depending on $n$, such that, for any Borel subset $V \subset Q(0,1)$ one has $|Q(0,1) \backslash V| \leq q_{0}$. Then we can find a constant $K_{0}>0$, only depending on $n$ and $x_{\infty} \in Q_{1 / 8} \cap V$ such that, for any $r \in\left(0, \frac{3}{4}\right)$,

$$
\left|Q\left(x_{\infty}, r\right) \backslash V\right| \leq K_{0}\left|Q_{1} \backslash V\right|^{\frac{1}{2}} \cdot \mathbb{R}^{n}
$$

Now we construct $b$ and $X$. We consider the following local dynamics. For a finite stopping time $T$ and two $F_{t}$-measurable random variables $N: \Omega \rightarrow Z$ and $J_{0}=\Omega \rightarrow \mathbb{R}^{n}$, we define the drift $b_{t}^{T, J_{0}, N}=\delta^{-2 N}\left(x_{\infty}-J_{0}\right), T \leq t \leq T+\delta^{2 n}$. For a smooth function $\psi: R \rightarrow[0,1]$, matching 1 on $\left(-\infty, \frac{\alpha}{2}\right]$ and vanishing on $[\alpha,+\infty)$, we solve the SDE

$$
J_{t}^{T, J_{0}, N}=J_{0}+\int_{T}^{t} \psi\left(\lambda\left(J_{s}^{T, J_{0}, N}\right)\right) b_{s}^{T, J_{0}, N} d s+\int_{T}^{t} \omega\left(J_{s}^{T, J_{0}, N}\right) \sigma\left(J_{s}^{T, J_{0}, N}\right) d W_{s}, \quad T \leq t \leq T+\delta^{2 N} .
$$

We define $\left(X_{t}\right)_{t \geq 0}$ as follows. Let $T_{0}=0$ be an initial time, $X_{0} \in Q\left(x_{\infty}, \frac{1}{4}\right)$ as so $x_{\infty}$ and $X_{0}$ are in $Q_{1 \backslash 8}$. We take $X_{0} \neq x_{\infty}$. Then there exists a random integer number $n_{0}$ such that $X_{0} \in Q\left(x_{\infty}, \delta^{n_{0}}\right) \backslash Q\left(x_{\infty}, \delta^{n_{0}+1}\right)$. Now we set $T_{1}=\delta^{2 n_{0}}$ and $X_{t}=J_{t}^{0, X_{0}, n_{0}}$ for $t \in\left[0, T_{1}\right]$. In another case $X_{0}=x_{\infty}$ then we take $b_{t}=0$ for $t \geq 0$ and $\left(X_{t}\right)_{t \geq 0}=S_{X_{0}}(0, \sigma)$. In this case we set $T_{k+1}=\infty$ and $n_{k}=+\infty, \forall k \geq 0$. The construction is over.

Since it is an initialization, we stop later by one step. Let $n_{0}<+\infty$ and $X_{T_{1}}=x_{\infty}$. We take $b_{t}=0$ for $t \geq T_{1}$ and define $\left(X_{t}\right)_{t \geq T_{1}}$ as the solution of

$$
X_{t}=X_{T_{1}}+\int_{T_{1}}^{t} \omega\left(X_{s}\right) \sigma\left(X_{s}\right) d W_{s}
$$

for $t \geq T_{1}$. In this case we set $T_{k+1}=\infty, n_{k}=\infty$ for $\forall k \geq 1$. The construction is over.

Now we are doing some iteration. Let $n_{0}<\infty, X_{T_{1}} \neq x_{\infty}$. Then there exists a random number $n_{1}$ such that $X_{T_{1}} \in Q\left(x_{\infty}, \delta^{n_{1}}\right) \backslash Q\left(x_{\infty}, \delta^{n_{1}+1}\right)$. Then for $t \in\left[T_{1}, T_{2}\right]$ we put $T_{2}=$ $T_{1}+\delta^{2 n_{1}}, X_{t}=J_{t}^{T_{1}, X_{T_{1}}, n_{1}}$. Now we apply stop later one step to $X_{T_{2}}$. We take, in the case $X_{T_{2}}=x_{\infty}, b_{t}=0$ at $t \geq T_{2}$ and define $\left(X_{t}\right)_{t \geq T_{2}}$ as the solution of

$$
X_{t}=X_{T_{2}}+\int_{T_{2}}^{t} \omega\left(X_{s}\right) \sigma\left(X_{s}\right) d W_{s}
$$

and take for any $k \geq 2 T_{k+1}=\infty, n_{k}=\infty$. The construction is over.

Now we do some iteration. It is clearly, the random times $\left(T_{k}\right)_{k \geq 0}$ are stopping times, i.d. $T_{k+1}$ is $F_{T_{k}}$ measurable. Let $S$ be an exit time of $X$ from the hypercube $Q\left(x_{\infty}, \frac{3}{4}\right)$ and we also introduce stopping times $\tau_{1}, \tau_{2}$. These are discrete stopping times for filtration $\left(F_{T_{k}}\right)_{k \geq 0}$. With $k \geq \tau_{1}$, if $t \in\left[T_{k}, T_{k+1}\right)$, then $d X_{t}=\omega\left(X_{t}\right) \sigma\left(X_{t}\right) d W_{t}$ and with $0 \leq k<\tau_{1}, T_{k+1}=T_{k}+\delta^{2 n_{k}}$, $d X_{t}=\delta^{-2 n_{k}} \psi\left(\lambda\left(X_{t}\right)\right)\left(x_{\infty}-X_{T_{k}}\right) d t+\omega\left(X_{t}\right) \sigma\left(X_{t}\right) d W_{t}$. For stopping time the parameter $\tau_{2}$ permits to evaluate the noise inside the system. Our investigation shows that in time less than $S$ there is enough noise in the system. $X$ hits $V$ before leaving the $Q\left(x_{\infty}, \frac{3}{4}\right)$. 
The reason why we expect such a behavior may be explained as follows.

Later we study growth of $\left(n_{k \wedge \tau}\right)_{k>0}$ up to exit time. For this goal we used stochastic comparison. We note that for any $0 \leq k<\tau_{1}$

$$
n_{k+1}=\infty \Rightarrow X_{T_{k+1}}=x_{\infty}, n_{k+1}=l \Rightarrow \delta^{l+1} \leq\left\|X_{T_{k+1}}-x_{\infty}\right\|<\delta^{l},
$$

$l$ is integer. Then for $0 \leq k<\tau$ and $T_{k}<S$ from above we deduce, that for $l \geq 0,\left\{n_{k+1}=\right.$ $\left.n_{k}-l\right\}=\left\{\delta^{n_{k}-l+1} \leq\left\|X_{T_{k+1}}-x_{\infty}\right\|<\delta^{n_{k}-l}\right\}$. We compare the conditional distribution of $n_{k+1}-n_{k}$ knowing $F_{T_{k}}$ with the distribution of some variable $\xi_{k+1}$ with values into integer $l \leq 1$ such that

$$
Q\left\{\xi_{k+1}=-l\right\}=\delta^{2(1+l)}, l \geq 0, Q\left\{\xi_{k+1}=1\right\}=1-\frac{\delta^{2}}{\left(1-\delta^{2}\right)} .
$$

Later we get deviation inequality. We have for $\delta \in\left(0, \delta_{1}\right)$ and any $k \geq 1$

$$
P_{0}\left\{n_{k} \leq \frac{k}{2}, \tau>k, T_{k}<S\right\} \leq \delta^{\frac{k}{2}}
$$

Now we evaluate the exit time $S$, so that

$$
S \leq T_{k+1} \Rightarrow \sup _{T_{k} \leq t \leq T_{k+1}}\left[\delta^{-n_{k}} \int_{T_{k}}^{t} 1_{\left\{\lambda\left(X_{S}>\alpha \backslash 2\right)\right\}} d s+\left|\int_{T_{k}}^{t} \omega\left(X_{s}\right) \sigma\left(X_{s}\right) d W_{s}\right|\right] \geq\left(\frac{2}{3}-\delta^{n_{k}}\right) .
$$

By Doob's maximal inequality we obtain on the event $\left\{T_{k \wedge \tau}<S\right\}$ at $k \geq 0$

$$
P_{T_{k \wedge \tau}}\left\{S \leq T_{(k+1) \wedge \tau}\right\} \leq \delta^{2\left(n_{k}+2\right)}\left[\frac{2}{3}-\delta^{n_{k}}\right]^{-2} 1_{\{k<\tau\}} .
$$

So that, we get later some calculations, that

$$
P_{0}\left(\bigcap_{k \geq 0}\left\{n_{k \wedge \tau} \geq \frac{\left(n_{k \wedge \tau}\right)}{4}\right\}\right) \geq 1-o(1)
$$

To end, we set $R=1+\sum_{k>0} \delta^{k}$. Later we can choose $R_{0}$ in the final statement. Since $P_{0}\left\{\exists k \geq 0: S<T_{k \wedge \tau}\right\} \leq o(1)$, the exit time is greater than all the times $\left(T_{k \wedge \tau}\right)_{k \geq 0}$. If $\tau$ is infinite, then the process $\left(X_{t}\right)_{t \geq 0}$ converges to $x_{\infty} \in V$ in time less than $R$ on the event $\left\{\forall k \geq 0, n_{k} \geq \frac{k}{2}\right\}$.

Let $\tau$ be finite, there are two cases: $1: \tau=\tau_{1}$, then $X_{T}=x_{\infty}$, the process hits $x_{\infty}$ in time less than $R ; 2 \tau=\tau_{2}$, then the process hits $V$ with a non-zero conditional probability and the hitting time is less than $R$.

Later we consider cases $\tau=+\infty ; \tau=\tau_{1}<\infty ; \tau<\infty, \tau_{2}<\tau_{1}$; combining above cases together. We use the fact

$$
E_{T_{\tau}}\left[\int_{T_{\tau}}^{T_{\tau+1}} \lambda\left(X_{s}\right) \omega\left(X_{s}\right) d s\right] \geq \delta^{2\left(n_{\tau+2}\right)}
$$

also $P_{T_{\tau}}\left\{\exists t \in\left(T_{\tau}, T_{\tau+1}\right): X_{t} \in V\right\} \geq \varepsilon$.

We choose $\delta, V$ and use integrability of the drift. Then we have

$$
E_{0}\left[\int_{0}^{T_{\tau+1} \wedge S}\left\|b_{t}\right\|^{P} d t\right] \leq 1+\sum_{k \geq 0} E_{0}\left[\delta^{(2-P) n_{k}} 1_{k<\tau, T_{k<S}}\right] .
$$




\section{REFERENCES}

1. R. Bass, Diffusions and elliptic operators, Springer-Verlag, New-York, 1958.

2. G. Barles, F. Da Lio, Local $C^{0, \alpha}$ estimates for viscosity solutions of Neumann-type boundary value problems, J. Differential Equations, 225 (2006), 202-241.

3. S. Chanillo, R. Wheeden, Weighted Poincare and Sobolev inequalites, Nonlinear Analysis, V.107, 1986, 1151-1226.

4. V. Guliyev, T. Gadjiev, Galandarova Sh., Dirichlet boundary value problems for uniformly elliptic equations in modified local generalized Sobolev-Morrey spaces, Electron. J. Qual. Theory Differ. Equ., 71 (2017), 1-17.

5. T. Gadjiev, Sh. Galandarova, V. Guliyev, Regularity in generalized Morrey spaces of solutions to higher order nondivergence elliptic equations with VMO coefficients, Electron. J. Qual. Theory Differ. Equ., 55, 2019, 1-17.

6. M.G. Crandall, H. Ishii, P.-L. Lions, Uniqueness of viscosity solutions of Hamilton-Jacobi equations revisited, J. Math. Soc. Japan, 39 (1987), №4, 581-596.

7. E. De Giorgi, Sulla differenziabilita e l'analiticita della estremali degli integrali multipli regolari, Mem. Accad. Sci. Torino Cl. Sci. Fis. Mat. Natur., 3 (1957), 25-43.

8. P. Gilbarg, N. Trudinger, Elliptic partial differential equations of second order, Springer-Verlag, Berlin, 1983.

9. N. Krylov, M. Safanov, An estimate for the probability of a diffusion process hitting a set of positive measure, Dokl. Akad. Nauk SSSR., 245 (1979), 18-20.

10. N. Krylov, M. Safonov, A property of the solutions of parabolic equations with measurable coefficients, Izv. Akad. nauk SSSR. Ser. Mat., 44 (1980), 161-175.

11. O. Ladyzhenskaya, N. Uraltseva, V. Solonnikov, Linear and quasilinear equations of parabolic type, Amer. Math. Society, Providence, R.I., 1967.

12. J. Nash, Continuity of solutions of parabolic and elliptic equations, Amer. J. Math., 80 (1958), 931-954.

13. Serrin J., Local behavior of solutions of quasilinear equations, Acta Math., 111 (1964), 247-302.

14. E. Pardoux, S. Peng, Backward stohastic differential equations and quasilinear parabolic partial differential equations, Lecture Notes in Control and Information Sciences, V.176, Springer, Berlin, 1992.

T. S. Gadjiev ${ }^{1, a}$, Ya. Rustamov $^{2, c}$, S. Aliev $^{1, b}$, T. A. Maharramova ${ }^{1, d}$

${ }^{1}$ Institute of Mathematics and Mechanics of NAS of Azerbaijan

Baku, Azerbaijan,

${ }^{2}$ Institute of Control Systems of NAS of Azerbaijan

Baku, Azerbaijan

a tgadjiev@mail.az

b soltanaliyev@yahoo.com

${ }^{c}$ terlan56@mail.ru

$d$ tarlanmaharramova@yahoo.com 